\title{
The mutation rates of EGFR in non-small cell lung cancer and KRAS in colorectal cancer of Chinese patients as detected by pyrosequencing using a novel dispensation order
}

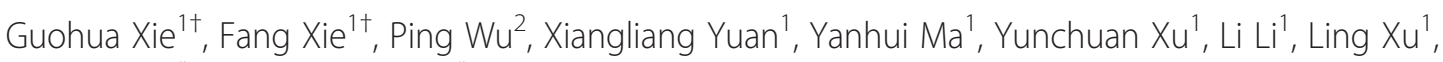
Ming Yang ${ }^{3^{*}}$ and Lisong Shen ${ }^{1^{*}}$

\begin{abstract}
Background: The purpose of this study was to develop a cost-effective approach for the determination of EGFR and KRAS mutations in formalin-fixed paraffin-embedded (FFPE) non-small cell lung cancer (NSCLC) and colorectal cancer (CRC) samples from Chinese patients based on a sensitive pyrosequencing (PS) technique.

Methods: The NSCLC and CRC cell lines were tested to determine the limitation of detection and reproducibility of the PS method. In addition, 494 NSCLC and 1099 CRC patient samples were assayed by PS to evaluate the EGFR or KRAS mutation patterns according to the clinicopathological features.

Results: The PS assay was able to reproducibly detect as few as $2 \%$ mutant alleles with excellent linearity. EGFR mutations were detected in $35.63 \%$ of the NSCLC samples, and KRAS mutations were detected in $39.76 \%$ of the CRC samples. EGFR mutations were more frequently observed to be significant by multivariate analysis in NSCLC patients who were 65 years old or younger $(O R=2.51)$, had a nonsmoking history $(O R=3.63)$, and adenocarcinoma $(O R=3.57)$, but not in females $(O R=0.64)$. KRAS mutations were more frequently detected in CRC patients who were female $(O R=1.64)$ and 50 years old or older $(O R=4.17)$, and had adenocarcinoma $(O R=2.41)$.

Conclusions: This is the first extensive validation of PS on FFPE samples using the detection of EGFR exons 18-21 mutations and KRAS exon 2 mutations. Our results demonstrate the utility of PS analysis for the detection of somatic EGFR and KRAS mutations in clinical samples and provide important clinical and molecular characteristics of NSCLC and CRC from Chinese patients.
\end{abstract}

Keywords: EGFR, KRAS, NSCLC, CRC, FFPE sample, Pyrosequencing

\section{Introduction}

Non-small cell lung cancer (NSCLC) and metastatic colorectal cancer (mCRC) are the most common cancers and the leading causes of cancer mortality [1]. Among the currently available therapeutic options, chemotherapy

\footnotetext{
* Correspondence: yangming_xh@163.com; lisongshen@yeah.net ${ }^{\dagger}$ Equal contributors

${ }^{3}$ Department of Anorectal Surgery and Colorectal Cancer Center, Xinhua Hospital, Shanghai Jiao Tong University School of Medicine, 1665 Kong Jiang Road, Shanghai 200092, China

'Department of Clinical Laboratory, Xinhua Hospital, Shanghai Jiao Tong University School of Medicine, 1665 Kong Jiang Road, Shanghai 200092, China

Full list of author information is available at the end of the article
}

for NSCLC and mCRC has been only marginally effective. Therefore, the development of novel and effective therapy has been and continues to be imperative for public health. In the past decade, molecularly targeted therapeutics has been developed for the treatment of advanced NSCLC and mCRC. Some small molecule tyrosine kinase inhibitors (TKIs) and anti-epidermal growth factor receptor (EGFR) monoclonal antibodies (mAbs) have been shown to be effective both in preclinical and clinical trials. TKIs targeting a mutant EGFR and mAbs binding to the extracellular domain of EGFR have shown significant benefit in the clinic, but their efficacy depends on the mutations of EGFR in the tyrosine kinase region and

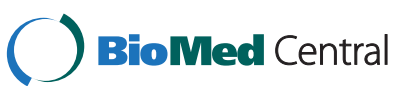


wild-type V-Ki-ras2 Kirsten rat sarcoma viral oncogene homolog (KRAS) in the EGFR signaling pathway, respectively, which have become established predictive markers for the stratification of NSCLC and MCRC patients for targeted treatment [2,3]. Accordingly, the US Federal Drug Administration has approved several TKIs and mAbs as anti-EGFR treatment for advanced NSCLC patients with the EGFR-activating mutation and for mCRC patients with wild-type KRAS, but not for NSCLC patients with wild-type EGFR or CRC patients with mutant KRAS $[4,5]$. Thus, accurate incidence rates of EGFR and KRAS mutations are critical to reckon the effectiveness of molecular-targeted agents as personalized treatment for advanced NSCLC and mCRC patients in any given population.

During the past decade, the incidences of the EGFR mutation in NSCLC patients and the KRAS mutation in CRC patients have been shown to vary across different ethnicities. The mutation rate of EGFR in NSCLC patients is $16-18 \%$ in North Americans and Europeans [6, 7], $19 \%$ in African-Americans [8], $22 \%$ in Indians [9], $29 \%$ in Koreans [10], and $40 \%$ in Japanese [11]; for the KRAS mutation in CRC patients, the rate is $37-54 \%$ in North Americans and Europeans, $47 \%$ in African-Americans [12], $24 \%$ in Indians [13], $27 \%$ in Koreans [12], and $38 \%$ in Japanese [14], as summarized in Additional file 1: Table S1. However, data regarding the frequencies of EGFR and KRAS mutations in a Chinese population currently remain contradictory and confusing. The currently available data show that the frequency of NSCLC EGFR mutations in patients from mainland China varies from 19 to $56 \%$ [9, 15-17], and the KRAS mutation rate in Chinese patients with CRC is 20-62 \% [18-20]. Moreover, the incidence of EGFR and KRAS mutations might be underestimated or overestimated because of clinically selected cases and the small sample sizes in those studies. Thus, it is of high importance to accurately determine the mutation rates of EGFR in NSCLC and KRAS in CRC with a large cohort of patients.

In addition, with the advent of personalized medicine, there is an urgent need for routine methods for rapid and accurate detection of changes of nucleic acid in clinical specimens. A wide range of techniques exist for mutation detection, of which dideoxy sequencing has been the gold standard [21]. However, the limited sensitivity and long turnaround time of these available methods have prompted the development of alternative techniques for routine clinical testing that have greater diagnostic practicality [21, 22]. Pyrosequencing is one of the latest methods that uses luminometric instead of electrophoretic detection [23]. This technique enables characterization of mutations and quantification with high accuracy of mutated alleles in samples with a low tumor cell density. Pyrosequencing is particularly suitable for the targeted sequencing of short DNA fragments amplified from older and less optimal tissue samples [24]. Owing to its high sensitivity, pyrosequencing seems to present a more reliable approach, allowing rapid, accurate, and high-throughput detection of a minimal fraction of mutated cells in archived clinical tumor tissue [25].

In this study, we designed an accurate and reliable pyrosequencing assay to determine the EGFR mutation in 494 NSCLC patients and the KRAS mutation in 1099 CRC patients of Chinese ethnicity. We further retrospectively analyzed and correlated the EGFR and KRAS mutations across different variables including age, gender, smoking status, and histology groups.

\section{Materials and methods}

\section{Cell lines and known mutated formalin-fixed paraffin-embedded (FFPE) tissues}

Six human NSCLC and CRC cell lines (Additional file 1: Table S2) and four known EGFR and KRAS mutationpositive FFPE tumor tissues (Additional file 1: Table S3) were initially used to validate the accuracy of the established pyrosequencing method. All the cell lines were purchased from the Cell Bank of the Chinese Academy of Sciences (Shanghai, China). The four known mutationpositive FFPE tissues with an expected mutation profile based on previous Sanger sequencing analysis were histologically confirmed to be tumor cells according to the hematoxylin-eosin-stained slides.

\section{Collection of patient samples}

NSCLC FFPE tissue specimens $(n=494)$ and CRC FFPE tissue specimens $(n=1099)$ were collected from the Department of Pathology of Xinhua Hospital affiliated to Shanghai Jiao Tong University School of Medicine (Shanghai, China) between January 2009 and December 2012. Written informed consent was obtained from all patients, and the study was approved by the Ethics Committee of Xinhua Hospital. Patient and tumor characteristics, such as age, gender, smoking status, histology and tumor sample type, are summarized in Table 1.

The material available for all tumors was tissue blocks. Before DNA extraction, representative sections were stained with haematoxylin and eosin (H\&E) and tumors were reviewed by two pathologists (LS and MY) and histologically classified according to the 2004 WHO criteria. Moreover, the percentages of tumor cells and extracellular mucin, if there was a relevant amount (more than $50 \%$ of the tumor), or lymphocyte inflammation (more than $10 \%$ of lymphocytes at $20 \times$ magnification) were assessed. Macrodissection was performed to guarantee at least $30 \%$ tumor in all cases in which there was sufficient material available for analysis. 
Table 1 Patient characteristics

\begin{tabular}{|c|c|c|c|}
\hline Variable & Number (\%) & Variable & Number (\%) \\
\hline NSCLC & $494(100)$ & CRC & $1099(100)$ \\
\hline Age (years) & & Age (years) & \\
\hline Mean & 64.70 & Mean & 60.95 \\
\hline Range & $38-85$ & Range & $36-82$ \\
\hline Gender & & Gender & \\
\hline Male & 334 (67.61) & Male & 657 (59.78) \\
\hline Female & 160 (32.39) & Female & $442(40.22)$ \\
\hline Smoking history & & Sublocalization & \\
\hline Ever smoker & $348(70.45)$ & Proximal colon & 373 (33.94) \\
\hline Never smoker & $146(29.55)$ & Distal colon & 355 (32.30) \\
\hline Pathological stage & & Rectum & 371 (33.76) \\
\hline I & $96(19.43)$ & Dukes' stage & \\
\hline$\|$ & 75 (15.18) & $A$ & $97(8.83)$ \\
\hline III & $132(26.72)$ & B & $517(47.04)$ \\
\hline IV & 185 (37.45) & C & $343(31.21)$ \\
\hline Unknown & $6(1.21)$ & D & $142(12.28)$ \\
\hline Pathology & & Pathology & \\
\hline$A D C$ & 310 (62.75) & $A D C$ & 1045 (95.09) \\
\hline SCC & $172(34.82)$ & SCC & $21(1.91)$ \\
\hline LCC & $6(1.21)$ & ASC & $9(0.82)$ \\
\hline ASC & $4(0.81)$ & UDC & $18(1.64)$ \\
\hline Others & $2(0.40)$ & Others & $6(0.55)$ \\
\hline Differentiation & & Differentiation & \\
\hline Poor & 97 (19.64) & Poor & 256 (23.29) \\
\hline Moderate & $249(50.40)$ & Moderate & $752(68.43)$ \\
\hline Well & $145(29.35)$ & Well & $78(7.10)$ \\
\hline Unknown & $3(0.61)$ & Unknown & $13(1.18)$ \\
\hline
\end{tabular}

NSCLC non-small cell lung cancer, $C R C$ colorectal cancer, $A D C$ adenocarcinoma, SCC squamous cell carcinoma, LCC large cell carcinoma, ASC adenosquamous carcinoma, UDC undifferentiated carcinoma

\section{DNA extraction}

The genomic DNA of the human cell lines was extracted using the QIAamp DNA Mini Kit (Qiagen, Germany). The genomic DNA of the FFPE samples was extracted using the GTpure ${ }^{\mathrm{TM}}$ FFPE Tissue Kit (GeneTech, China). All of the extracted DNA samples were quantitated with NanoDrop spectrophotometer 2000c (Thermo Fisher, USA) and stored at $-20{ }^{\circ} \mathrm{C}$ until use.

\section{Cell-mixing studies}

To analyze the assay linearity and sensitivity, we performed reconstruction experiments with the NSCLC cell lines and CRC cell lines. The NSCLC or CRC cell lines harboring heterozygous or homozygous mutations were serially diluted with the corresponding wild-type cells. The proportions of the mutant cell lines were adjusted to $100 \%, 50 \%, 30 \%, 20 \%, 10 \%, 5 \%, 3 \%, 2 \%$, and $0 \%$ (mutant-type:wild-type), respectively. The genomic DNA extracted from each dilution was subjected to subsequent pyrosequencing and dideoxy sequencing on three separate, consecutive days. The actual percentage of the mutant allele was determined by pyrosequencing data from undiluted tumor cell DNA. For each tumor cell dilution, a theoretical percentage of the mutant allele was then calculated.

\section{Mutation analysis by pyrosequencing and direct dideoxy sequencing}

The polymerase chain reaction (PCR) and pyrosequencing primers are listed in Additional file 1: Table S4. The PCR amplicons for pyrosequencing were bound to Streptavidin-Sepharose HP (GE Healthcare, USA), purified, washed, denatured with $0.2 \mathrm{M} \mathrm{NaOH}$, and washed again. The pyrosequencing primer $(0.3 \mu \mathrm{M})$ was annealed to the purified single-stranded PCR product, the pyrosequencing assay was performed on a PyroMark Q24 system (Qiagen, Germany), following the manufacturer's instructions. The dispensation order for EGFR exons 18, 19, 20, and 21 was GCAAGTGCTGATGCTCGT, ACGAATAC GACGACGCTAACAGTCTACGA, GACTAGCAGCTG CATGCT, and TGCGTGTCAACTACG, respectively. The dispensation order for the KRAS was TACGACTCA GATCGTAG. Our dispensation order was longer than that found in the commercial kit; additional bases were introduced in strategic positions, according to possible expected mutated sequences (Additional file 1: Figure S1). The amplicons for direct Sanger sequencing were analyzed and purified with the GTpure ${ }^{\mathrm{TM}}$ Gel/PCR Extraction Kit (GeneTech), and sequenced on an ABI 310 genetic analyzer (Applied Biosystems).

\section{Statistical analysis}

All statistical analyses were performed using SAS ver. 9.2 (SAS Institute, Cary, NC). For continuous variables, the median and range were calculated. A $\chi^{2}$ test was performed in a univariate analysis in order to investigate the relationship between the EGFR or KRAS gene mutation rate and patient background factors. Based on the results, multivariate logistic regression was performed, and the odds ratio (OR) and $95 \%$ confidence interval (95\% CI) were calculated. Statistical significance was set at $p<0.05$.

\section{Results}

Pyrosequencing with novel nucleotide dispensation order accurately detects EGFR or KRAS mutations both in cultured cells and FFPE tissues

The established pyrosequencing method was able to discriminate the wild-type from the different mutations presented in the mutant cell lines. As seen in the pyrosequencing traces of the targeted sequence of the six cell lines, the NSCLC cell lines H1650 (c.2235_2249del15, 
Fig. 1aiii) and H1975 (c.2573T>G, Fig. 1aiiii) showed specific peak patterns, which were readily distinguishable from that of the EGFR exons 19 and 21 wild-type NSCLC cell line A549 (Fig. 1ai and ii). Similarly, the A549 (KRAS exon 2 c.34G>A, Fig. 1bii) and the CRC cell lines SW480 (KRAS exon 2 c.35G $>$ T, Fig. 1biii) and DLD-1 (KRAS exon 2 c.38G >A, Fig. 1biiii) showed specific pyrogram traces, which were readily distinguishable from that of the KRAS exon 2 wild-type CRC cell line HT-29 (Fig. 1bi). The designed pyrosequencing assay was further tested with the known mutant NSCLC- and CRC-FFPE tissues (Additional file 1: Table S3). The two types of NSCLCFFPE tissues harboring different EGFR TK domain mutations (Fig. 1ci and ii) and the two types of CRC-FFPE tissues with known KRAS exon 2 mutations (Fig. 1ciii and iiii) were detected by the established pyrosequencing analysis, respectively. Dideoxy sequencing confirmed the results of the pyrosequencing traces.

\section{Pyrosequencing with novel nucleotide dispensation order demonstrates high sensitivity and excellent linearity and reproducibility in detecting EGFR and KRAS mutations}

Assay sensitivity of the pyrogram profile in discriminating different percentages of mutated alleles was initially evaluated by using serial dilutions of mutated DNA, derived from a cultured cell line with a known EGFR or KRAS mutation, and variably mixed with wild-type DNA obtained from a wild-type cell line. We tested $100 \%, 50 \%, 30 \%, 20 \%, 10 \%, 5 \%, 3 \%, 2 \%$, and $0 \%$ (mutant-type/wild-type) dilutions of H1650, H1975, SW480, and DLD-1cell lines (Fig. 2a). Our results demonstrated that this method could detect at least $2 \%$ of the mutated alleles in the heterozygous mutant EGFR c.2235_2249del15 (Fig. 2ai) and homozygous mutant KRAS c.35G $>$ T (Fig. 2aiii), $3 \%$ of the mutated alleles in the heterozygous mutant EGFR c.2573T $>$ G dilution (Fig. 2aii), and $5 \%$ of the mutated alleles in the heterozygous mutant KRAS c.38G>A dilution (Fig. 2aiiii). Compared with the dideoxy sequencing tracings from the amplifications on day 1 , a small mutant peak was visible in the dideoxy sequencing tracings of the 15-20\% homozygous to $20-30 \%$ heterozygous mutant dilutions (Additional file 1: Figures S2-S5).

For linearity testing, based on the data generated from the separate NSCLC or CRC cell mixing analyses, a linear relationship between the actual and theoretical percentages of the mutant allele was identified (Fig. 2b, Additional file 1: Tables S5-S6). The coefficient of correlation (r) was 0.99 for EGFR exon 19 c.2235_2249del15 (G>A) (Fig. 2bi), KRAS exon 2 c.35G $>$ T (Fig. 2biii), and KRAS exon 2 c.38G $>$ A (Fig. 2biiii); the $\mathrm{r}$ value was 0.96 for EGFR exon 21 c.2573 T>G (Fig. 2bii), indicating an excellent linearity and reproducibility.

\section{Pyrosequencing analysis results of EGFR mutations in exons 18-21 in 494 NSCLC clinical samples}

After standardization and validation by direct DNA sequencing for the robustness, linearity, and sensitivity of our designed pyrosequencing method, we applied it as a routine clinical test for common EGFR mutation assay in 494 patients diagnosed with NSCLC (Table 1). Overall, $35.63 \%(176 / 494)$ of the NSCLC samples were identified to have EGFR TK domain mutations, representing 16 different nucleotide mutation types (Additional file 1: Figure S6). A total of $18.83 \%$ (93/ 494) of the mutations were located in exon 19, and $16.80 \%(83 / 494)$ were located in exon 21. A total of $52.27 \%(92 / 176)$ were small in-frame deletions in exon 19 , while $47.16 \%(83 / 176)$ were missense mutations in exon 21. The most common mutation in exon 19 was delE746-A750 (56.99 \%, 53/93). In exon 21 , the most common mutation was located on L848R (97.59 \%, 81/83). One rare exon 19 missense mutation (c.2239 TT>CC, p.L747P) was detected. EGFR exon 18 and exon 20 mutations were not detected in this cohort of NSCLC samples (Table 2).

\section{EGFR mutations and clinicopathological features of NSCLS patients}

Using univariate analysis, when all histologies were analyzed together, females showed a significantly higher frequency of the EGFR mutation compared with males ( $42.50 \%$ vs. $32.34 \%, p=0.028)$. Within the different age groups, the mutation rate was significantly higher in those 65 years old or younger than in those older than 65 years old $(45.02 \%$ vs. $24.22 \%, p<0.001)$. Those with ADC showed higher mutations than any other histology (45.48 \% vs. $19.02 \%, p<0.001)$. When the ADC subgroup was analyzed by gender, the mutation rate was predominantly higher among females compared to males $(56.12 \%$ vs. $40.57 \%, p=0.011)$. In addition, the smoking status was closely associated with the EGFR mutation rate, and the mutation rate was higher in the never-smoker group than in the smoker group $(50.68 \%$ vs. $29.31 \%, p<0.001$ (Table 3$)$. However, there was no difference in the EGFR mutation rate between males and females in the never-smoker group (52.38 \% vs. $50.40 \%, p=0.867)$. Similarly, no significant correlation of EGFR mutations between the neversmoker male or female ADC patients $(60.00 \%$ vs. $65.38 \%, p=0.69)$ was observed. When sex, age, histotype, and smoking history were tested by multivariate analysis against the presence of EGFR mutations as a dependent variable, only the female data did not remain significant. No significant associations between EGFR mutations and differentiation status or tumor stage were observed (Table 3). 

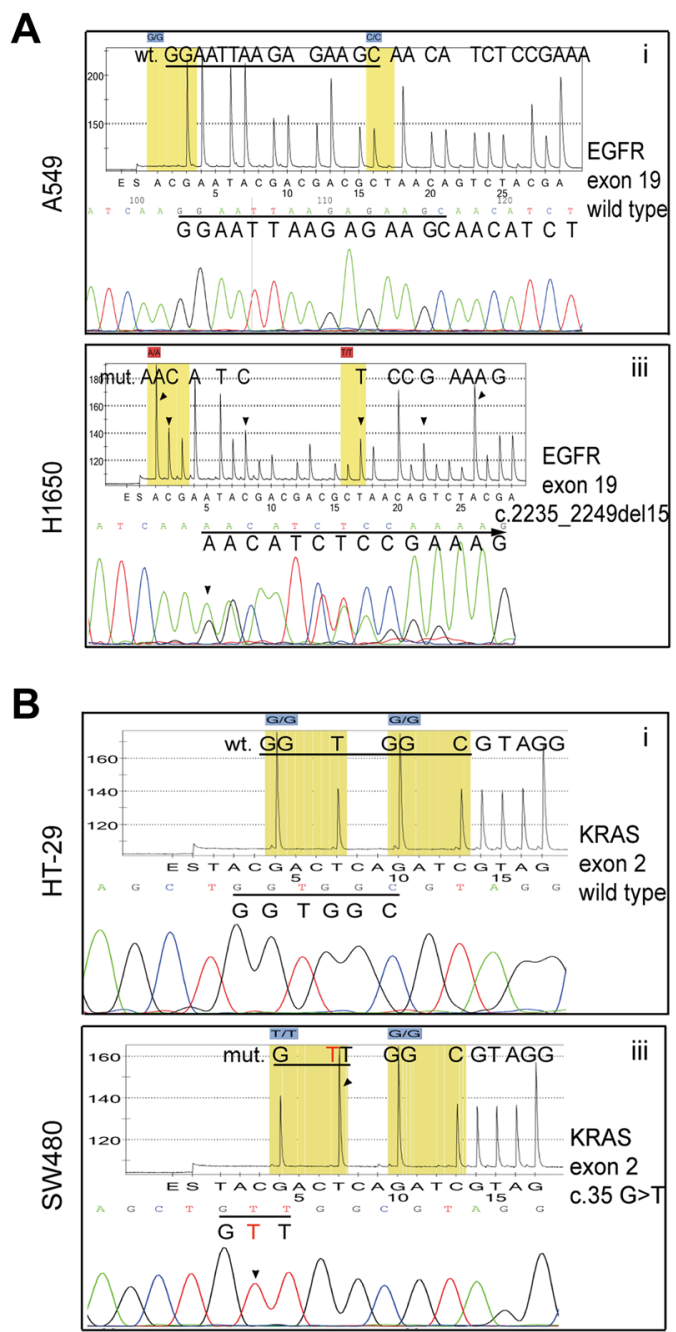

C

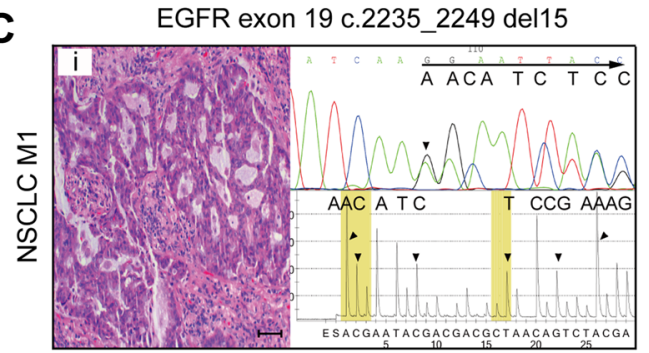

KRAS exon 2 c. 35 G>A

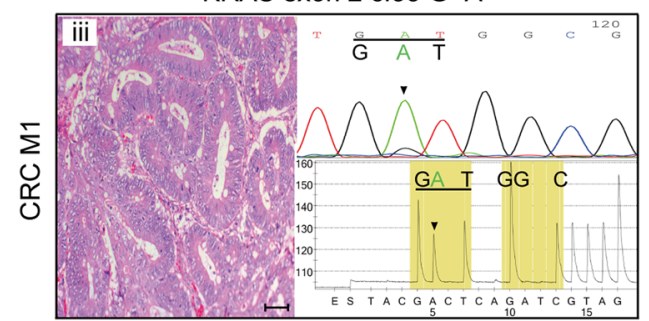

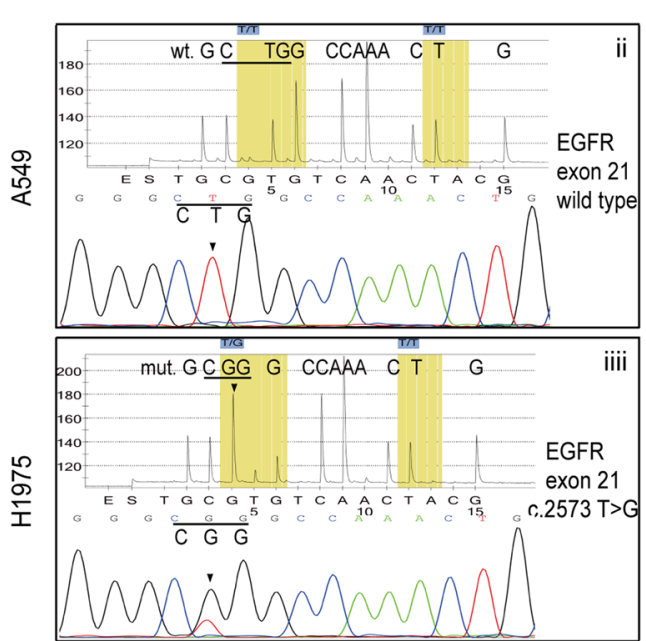
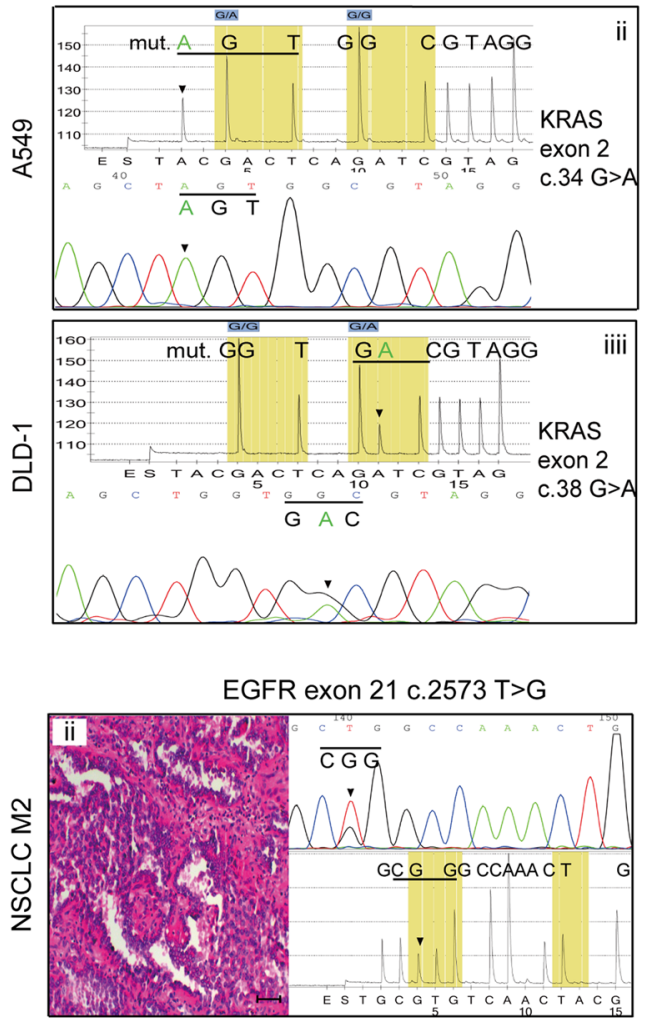

KRAS exon 2 c.38 G>A

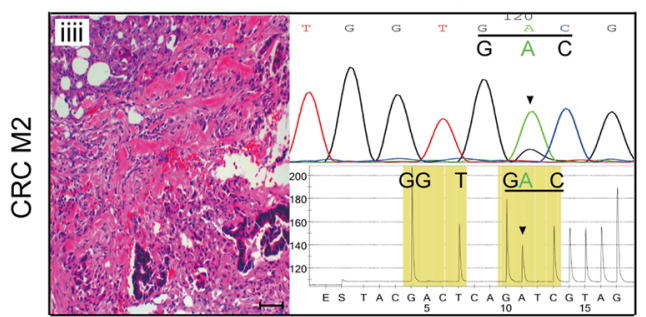

Fig. 1 (See legend on next page.) 
(See figure on previous page.)

Fig. 1 Validation of the established pyrosequencing analysis method using a novel dispensation order on cell lines and FFPE tissues. DNA from six tumor cell lines and four FFPE tissues was analyzed in parallel by pyrosequencing and dideoxy sequencing. a Examples of pyrograms obtained on the NSCLC cell lines A549 ( $i$ and ii), H1650 (iii), and H1975 (iiii) for mutational analysis of EGFR exon 19 or exon 21 . b Examples of pyrograms obtained on the CRC cell lines HT-29 (i), SW480 (iii), and DLD-1 (iiii) as well as the NSCLC cell line A549 (ii) for mutational analysis of KRAS exon 2 , codons 12 and 13. c To test the suitability of pyrosequencing for clinical samples, the assay was used to analyze EGFR or KRAS mutations in the following known mutated FFPE tissues: NSCLC M1 (i), NSCLC M2 (ii), CRC M1 (iii), and CRC M2 (iiii). All of the targeted mutations occurring in the cell lines and FFPE tumor tissues could easily be distinguished by pyrosequencing. The results for the six cell lines and four FFPE tissues were $100 \%$ concordant between the established pyrosequencing and dideoxy sequencing methods. The target nucleotide sequences are labeled and underlined. The horizontal axis of each pyrogram, from left to right, indicates the order of reagent addition. E represents enzyme. $\mathbf{S}$ represents substrate. Mutation points are indicated by light shading. The vertical axis represents the luminescence intensity, of which the peak heights are proportional to the number of each nucleotide incorporated at one time. Sites of variation are indicated by arrows. Data are representative of five independent analyses of the same sample

\section{Pyrosequencing analysis results of KRAS mutations in exon 2 in 1099 CRC clinical samples}

In 1099 Chinese CRC-FFPE samples (Table 1), a total of 437 missense mutations were detected at KRAS codons 12 and 13 (39.76\%, 437/1099). Nine types of KRAS exon 2 hotspot mutations were observed (Additional file 1: Figure S7). The mutation frequencies of exon 2, codon 12 and codon 13 were $30.30 \%(333 / 1099)$ and $9.46 \%$ (104/1099), respectively. The frequently observed mutations were $\mathrm{G}>\mathrm{A}$ transition (300/437, $67.65 \%$ ), followed by $\mathrm{G}>\mathrm{T}$ transversion (113/437, $25.86 \%$ ), and G>C transversion $(24 / 437,5.49 \%)$. Furthermore, $76.20 \%$ (333/437) of the mutations were located in codon 12 , in which the base substitutions were mainly located at the first and second nucleotides. The screening results also showed that six CRC patients harbored codon 12 double $\mathrm{G}>\mathrm{A}$ transitions (c.34G>A and c.35G>A, $1.37 \%, 6 / 437$ ). Meanwhile, 104 patients had detectable mutations at codon 13 $(23.80 \%, 104 / 437)$, and the $\mathrm{G}>\mathrm{A}$ transition at the second nucleotide was the most frequent mutant type (c.38G $>A$, $96.15 \%, 100 / 104$ ) (Table 2).

\section{KRAS mutations and clinicopathological features of CRC patients}

By univariate analysis, correlation of the KRAS mutation with clinicopathological data indicated that KRAS mutations were higher in females than in males $(46.15 \%$ vs. $35.46 \%, p<0.01$ ) (Table 4). Interestingly, KRAS mutations were significantly higher in patients who were older than 50 years old in comparison with patients younger than 50 years old ( $45.67 \%$ vs. $17.67 \%, p<0.001)$, indicating that KRAS mutations are uncommon in younger CRC patients. Moreover, KRAS mutations were more prevalent in ADC patients when compared to non-ADC patients such as those with SCC, ASC, and UDC (40.86 \% vs. $18.52 \%$, $p=0.002)$. However, KRAS mutations were not found to be significantly associated with moderately/poorly differentiated tumors in comparison with well-differentiated tumors. The logistic multivariate analysis results were in line with the univariate analysis results. No significant association between KRAS mutations and tumor sublocalization or the Dukes' stage was observed in these CRC patients (Table 4).

\section{Discussion}

In the present study, we established a practical and reliable pyrosequencing assay using novel nucleotide dispensation order for the detection of EGFR and KRAS mutations in a large cohort of Chinese NSCLC and CRC patients. We further retrospectively analyzed the actual incidence of genetic abnormalities of EGFR and KRAS and their distribution according to clinicopathological features in Chinese patients.

In our analysis of 494 NSCLC cases, the EGFR mutation rate was $35.63 \%$ in the Chinese population, which was significantly higher than the EGFR mutation rates reported in western countries, but was similar to that of other Eastern populations (Additional file 1: Table S1). We found a higher rate of the EGFR mutation in ADC patients than in all patients (45.48\% vs. $35.63 \%)$ and among females compared to males (42.50 \% vs. $32.34 \%$ ), similar to rates in Indian, Korean, and Japanese patients $[10,11,26]$. Although the mutation rate varies significantly between ever smokers and never smokers across different ethnicities, EGFR mutations have been consistently reported to be more common in never smokers as compared to ever smokers [9-11, 16, 27]. Consistently, we found that the mutation rates of EGFR for ever smokers and never smokers were 50.68-29.31\%, respectively. Histopathologically, the mutation rates among females with ADC were predominantly higher than in males with ADC (56.12 \% vs. $40.57 \%)$, consistent with previous studies [28, 29]. However, no significant differences were observed between never-smoker ADC males and females, indicating that the lack of a gender bias among never smokers was possibly due to the fact that there was a lower proportion of nonsmoking males (21 nonsmokers out of 334 males) than nonsmoking females (125 nonsmokers out of 160 females) in this study. These findings are also consistent with similar studies on females of other Asian ethnicities who never smoked, wherein the EGFR mutation rate varies with the clinical stage of the female 
A

A EGFR exon 19 c.2235_2249 del15 (G>A)

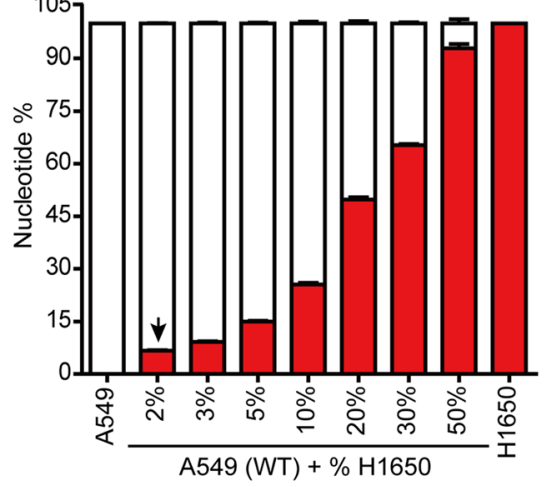

口 G

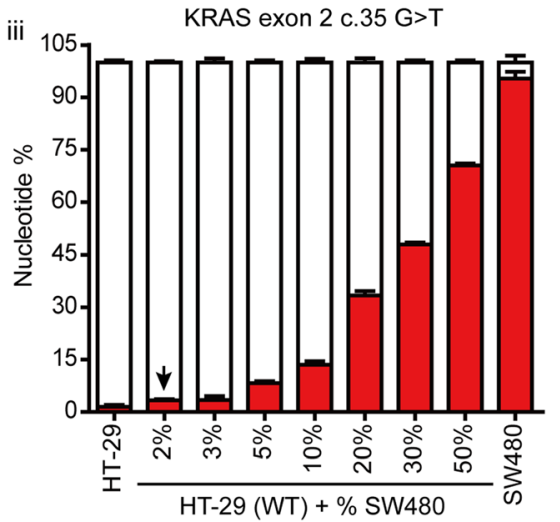

B

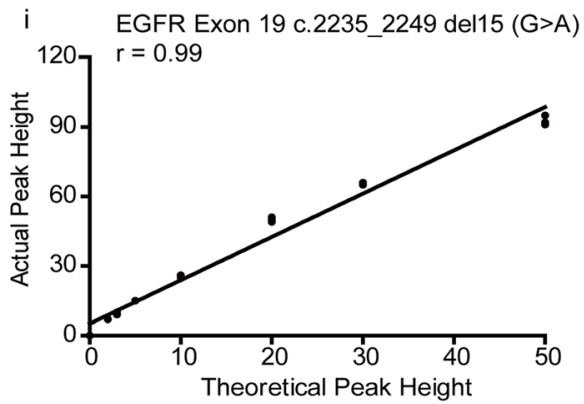

iii

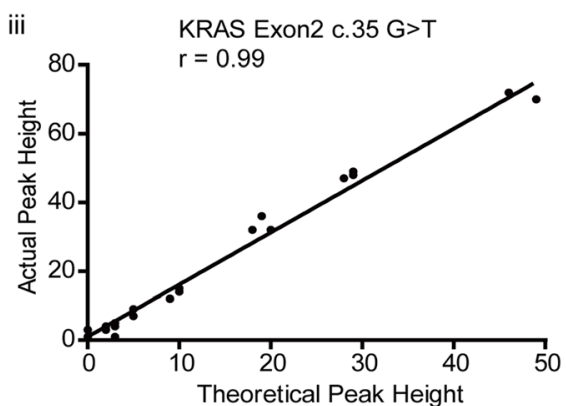

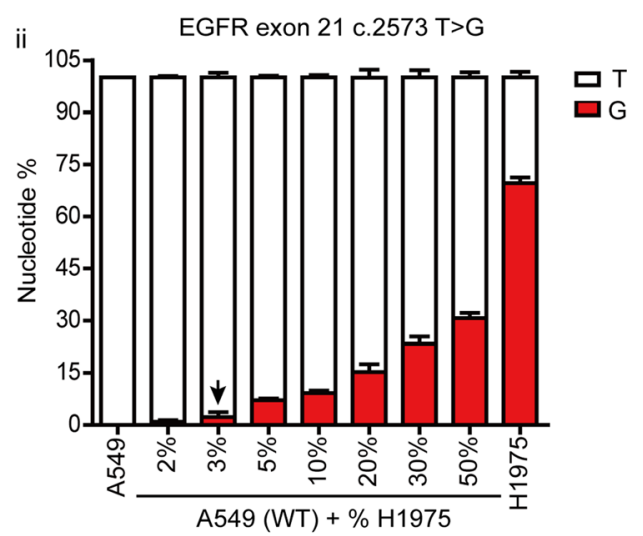

iiii
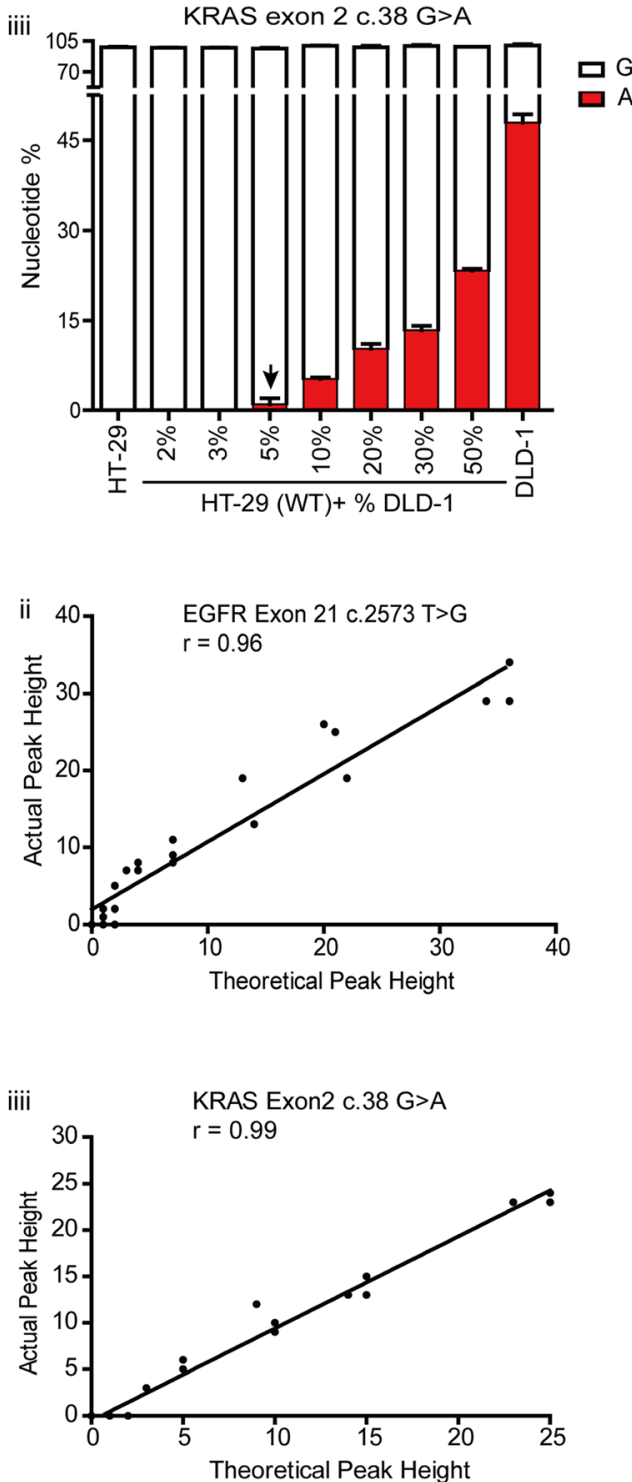

Fig. 2 (See legend on next page.) 
(See figure on previous page.)

Fig. 2 Limit of detection and assay linearity. Mixtures of DNA from EGFR or KRAS cell lines were analyzed in triplicate and in parallel by pyrosequencing and dideoxy sequencing methods to analyze the limit of detection and assay linearity (Additional file 1: Figures S2-S5, Tables S5-S6). a To assess the limit of detection, cell line DNA was mixed to produce samples containing differing proportions of mutant alleles. The established pyrosequencing method allows for $2 \%$ of EGFR exon 19 c.2235_2249del15 mutant alleles (i), 3 \% of EGFR exon21 c2573 T>G mutant alleles (ii), 2 \% of KRAS exon2 c.35G>T mutant alleles (iii), and $5 \%$ of KRAS exon 2 c.38G >A mutant alleles (iiii) to be detected with confidence. The percentages on the horizontal axis indicate the calculated percentages of mutant alleles present, while the vertical axis indicates the percentage of nucleotide. The white column represents the percentage of detected wild-type nucleotides; the red column within the white column represents the percentage composition of the detected mutant nucleotides. Arrows indicate the DNA dilutions in which mutations could be reliably detected above background noise. WT means wild-type. $\mathbf{b}$ To assess the assay linearity of pyrosequencing, theoretical peak heights, calculated from the initial mutant allele peak percentage in undiluted mutant cells, were correlated with actual peak heights generated for each dilution. For mutational analysis of EGFR exon 19 c.2235_2249del15 (i), KRAS exon 2 c35 G>T (iii), and c.38 G>A (iiii), Pearson's correlation ( $r$ )= 0.99; for mutational analysis of EGFR exon 21 c.2573 T>G (ii), $r=0.96$, indicating a linear relationship. A, adenine; $G$, guanine; $T$, thymine; C, cytosine. Data are from three independent experiments

Table 2 Summary of EGFR exons 18-21 mutations in 494 NSCLC-FFPE tissues and KRAS exon 2 mutations in 1099 CRC-FFPE tissues as detected by our designed pyrosequencing method

\begin{tabular}{|c|c|c|c|c|c|}
\hline Gene & Exon & $\begin{array}{l}\text { Type of } \\
\text { mutation }\end{array}$ & $\begin{array}{l}\text { Nucleotide } \\
\text { change }\end{array}$ & $\begin{array}{l}\text { Amino acid } \\
\text { change }\end{array}$ & $\begin{array}{l}\text { No. with } \\
\text { mutation (\%) }\end{array}$ \\
\hline \multirow[t]{16}{*}{ EGFR } & 19 & Missense & c.2239TT>CC & p.L747P & $1(0.20)$ \\
\hline & \multirow[t]{13}{*}{19} & \multirow[t]{13}{*}{ Deletion } & c.2235_2249del15 & p.E746_A750 & $53(10.73)$ \\
\hline & & & c.2236_2250del15 & p.E746_A750 & $18(3.64)$ \\
\hline & & & c.2237_2251del15 & p.E746_T751 & $2(0.40)$ \\
\hline & & & c.2237_2253del17 & p.E746_T751 & $1(0.20)$ \\
\hline & & & c.2237_2255del19 & p.E746_S752 & $1(0.20)$ \\
\hline & & & c.2237_2257del21 & p.E746_P753 & $1(0.20)$ \\
\hline & & & c.2239_2248del10 & p.L747_E749 & $2(0.40)$ \\
\hline & & & c.2239_2253del15 & p.L747_T751 & $1(0.20)$ \\
\hline & & & c.2239_2256del18 & p.L747_S752 & $2(0.40)$ \\
\hline & & & c.2240_2254del15 & p.L747_T751 & $2(0.40)$ \\
\hline & & & c.2240_2257del18 & p.L747_P753 & $5(1.01)$ \\
\hline & & & c.2253_2276del24 & p.S752_1759 & $3(0.61)$ \\
\hline & & & c.2254_2277del24 & p.S752_1759 & $1(0.20)$ \\
\hline & \multirow[t]{2}{*}{21} & \multirow[t]{2}{*}{ Missense } & c. $2573 \mathrm{~T}>\mathrm{G}$ & p.L858R & $81(16.40)$ \\
\hline & & & c. $2582 \mathrm{~T}>\mathrm{A}$ & p.L861Q & $2(0.40)$ \\
\hline Total & & & & & $176(35.63)$ \\
\hline \multirow[t]{9}{*}{ KRAS } & \multirow[t]{9}{*}{2} & \multirow[t]{9}{*}{ Missense } & $c .34 G>T$ & p.G12C & $24(2.18)$ \\
\hline & & & c.34G $>\mathrm{A}$ & p.G12S & $26(2.37)$ \\
\hline & & & c. $34 \mathrm{G}>\mathrm{C}$ & p.G12R & $1(0.09)$ \\
\hline & & & c. $35 \mathrm{G}>\mathrm{A}$ & p.G12D & 168 (15.29) \\
\hline & & & c. $35 \mathrm{G}>\mathrm{C}$ & p.G12A & $23(2.09)$ \\
\hline & & & c.35G $>T$ & p.G12V & 85 (7.73) \\
\hline & & & $\begin{array}{l}c .34 G>A+ \\
c .35 G>A\end{array}$ & $\begin{array}{l}\text { p.G12S + } \\
\text { p.G12D }\end{array}$ & $6(0.55)$ \\
\hline & & & $c .37 G>T$ & p.G13C & $4(0.36)$ \\
\hline & & & c.38G $>\mathrm{A}$ & p.G13D & $100(9.10)$ \\
\hline Total & & & & & 437 (39.76) \\
\hline
\end{tabular}

EGFR epidermal growth factor receptor, KRAS V-Ki-ras2 Kirsten rat sarcoma viral oncogene homolog, NSCLC non-small cell lung cancer, CRC colorectal cancer, FFPE formalin-fixed paraffin-embedded never-smoker patients [30-32]. For lung SCC, there have been few studies focusing on Asians, with conflicting results. Previous studies have shown that the EGFR mutation rates of South Korean, Indian, and Japanese patients were $0.96 \%$ (1/104), $3.88 \%$ (4/103), and $6.50 \%(8 / 123)$ [33-35], respectively. Two Chinese-based studies reported EGFR mutation rates of $14.54 \%(41 / 282)$ and $29.73 \%$ $(11 / 37)[16,17]$. In the SCC cohort of this study $(n=172)$, we found that the EGFR mutation rate was $19.19 \%$ (33/172), similar to the previously reported Chinese-based data, implying that the EGFR mutation test for SCC needs to be considered as a routine practice.

Our retrospective analysis of 1099 samples from Chinese CRC patients showed that the frequency of a KRAS mutation was $39.76 \%$, which is similar to the previously published data from Japan, the Netherlands, Germany, and the United States (37-43\%), but higher than those from Thailand, India, South Korea, Oman, and Australia (23-31 \%) (Additional file 1: Table S1). Except for the consideration of the sensitivity of the techniques used in some studies, the variations in the frequency of the KRAS mutation worldwide may be explained by the patient ethnicity and geographical distribution. Studies from western nations have reported KRAS G12D as the most recurrent transition, followed by G12V, G12C, G12S, and G12A [36, 37]. In our study, the corresponding order was G12D, G12V, G12S, G12C, and G12A. Among KRAS codon 13 mutations, G13D was the major mutation, followed by G13C and G13R or G13S in western populations [36, 37]. However, in the current study, predominantly only G13D and rarely G13C mutations were observed, while none of the cases showed G13R. These data suggest that there may be some racial differences in the patterns of KRAS mutations.

In agreement with previous studies [14, 19, 38], our results showed that KRAS mutations occurred more frequently in females than in males. Moreover, consistent with studies of India and Japan [13, 14], CRC patients older than 50 years old demonstrated significantly higher KRAS mutation rates than patients younger than 50 years old (396/867), indicating that the older the patient, the 
Table 3 Correlation of EGFR mutations with clinicopathological features of NSCLC patients

\begin{tabular}{|c|c|c|c|c|c|c|c|}
\hline \multirow[t]{2}{*}{ Variable } & \multirow[t]{2}{*}{ Number (\%) } & \multicolumn{2}{|l|}{ EGFR } & \multicolumn{2}{|l|}{ Univariate } & \multicolumn{2}{|l|}{ Multivariate } \\
\hline & & Mutation (\%) & Wild type (\%) & Odds ratio $(95 \% \mathrm{Cl}$ ) & $p$ value & Odds ratio (95 \% Cl) & $p$ value \\
\hline \multicolumn{8}{|l|}{ Gender } \\
\hline Male & $334(67.61)$ & $108(32.34)$ & $226(67.66)$ & Ref. & & Ref. & \\
\hline Female & 160 (32.39) & $68(42.50)$ & $92(57.50)$ & $1.55(1.05-2.28)$ & 0.028 & $0.64(0.32-1.28)$ & 0.209 \\
\hline \multicolumn{8}{|l|}{ Age } \\
\hline$\leq 65$ & $271(54.86)$ & $122(45.02)$ & 149 (54.98) & $2.56(1.73-3.78)$ & $<0.001$ & $2.51(1.65-3.81)$ & $<0.001$ \\
\hline$>65$ & $223(45.14)$ & $54(24.22)$ & 169 (75.78) & Ref. & & Ref. & \\
\hline \multicolumn{8}{|c|}{ Smoking history } \\
\hline Ever smoker & $348(70.45)$ & $102(29.31)$ & $246(70.69)$ & Ref. & & Ref. & \\
\hline Never smoker & $146(29.55)$ & $74(50.68)$ & $72(49.32)$ & $2.48(1.66-3.69)$ & $<0.001$ & $3.63(1.83-7.19)$ & $<0.001$ \\
\hline \multicolumn{8}{|l|}{ Never smoker } \\
\hline Male & $21(14.38)$ & $11(52.38)$ & $10(47.62)$ & Ref. & & & \\
\hline Female & $125(85.62)$ & $63(50.40)$ & $62(49.60)$ & $0.92(0.37-2.33)$ & 0.867 & & \\
\hline \multicolumn{8}{|c|}{ Pathologic stage } \\
\hline $1+\|$ & $171(34.62)$ & $55(32.16)$ & $116(67.84)$ & Ref. & & Ref. & \\
\hline$I I I+I V$ & $323(65.38)$ & $121(37.46)$ & $202(62.54)$ & $1.26(0.85-1.87)$ & 0.243 & $1.45(0.94-2.24)$ & 0.089 \\
\hline \multicolumn{8}{|l|}{ Pathology } \\
\hline Non-ADC & $184(32.75)$ & $35(19.02)$ & $149(80.98)$ & Ref. & & Ref. & \\
\hline ADC & $310(62.75)$ & $141(45.48)$ & $169(54.52)$ & $3.55(2.31-5.46)$ & $<0.001$ & $3.57(2.28-5.61)$ & $<0.001$ \\
\hline Male & 212 (68.39) & $86(40.57)$ & $126(59.43)$ & Ref. & & & \\
\hline Female & $98(31.61)$ & $55(56.12)$ & $43(43.88)$ & $1.87(1.15-3.04)$ & 0.011 & & \\
\hline \multicolumn{8}{|c|}{ Never smoker ADC } \\
\hline Male & 15 (16.13) & $9(60.00)$ & $6(40.00)$ & Ref. & & & \\
\hline Female & $78(83.87)$ & $51(65.38)$ & $27(34.62)$ & $1.26(0.41-3.91)$ & 0.69 & & \\
\hline \multicolumn{8}{|l|}{ Differentiation } \\
\hline Well & 145 (29.35) & $50(34.48)$ & $95(65.52)$ & Ref. & & Ref. & \\
\hline Moderate & $249(50.40)$ & $92(36.95)$ & $157(63.05)$ & $1.11(0.73-1.71)$ & 0.623 & $1.14(0.72-1.82)$ & 0.573 \\
\hline Poor & 97 (19.64) & $34(35.05)$ & $63(64.95)$ & $1.03(0.60-1.76)$ & 0.927 & $1.10(0.60-2.00)$ & 0.759 \\
\hline
\end{tabular}

EGFR epidermal growth factor receptor, NSCLC non-small cell lung cancer, ADC adenocarcinoma, Cl confidence interval, Ref. reference group

higher the KRAS mutation rate in CRC patients. Interestingly, our findings demonstrate that tumors with KRAS mutation tend to occur more frequently in ADC as compared to the non-ADC subtypes, which further supports the previous observation [13]. Other clinicopathological features including tumor differentiation, location, and staging did not show any association with KRAS mutation in our retrospective analysis.

Regarding the importance of EGFR and KRAS mutation detection for the prediction and prognosis of NSCLC and $\mathrm{CRC}$, a reliable diagnostic test may affect future therapeutic decision-making. In this study, we demonstrated that our designed pyrosequencing assay using a novel nucleotide dispensation order is a reliable and accurate technique for the detection of EGFR and KRAS hotspot mutations from FFPE tissues. First, pyrosequencing was established and validated in a set of DNA samples obtained from several cell lines and mutation-positive FFPE tumor tissues. The homozygous or heterozygous mutations in the NSCLC (Fig. 1a) and CRC cell lines (Fig. 1b) and mutant FFPE tissues (Fig. 1c) were accurately detected with this pyrosequencing method. Next, the sensitivity for detection of mutations by our designed pyrosequencing method was sufficiently high as it was able to detect mutations in samples containing as few as $2 \%$ homozygous to $5 \%$ heterozygous mutated alleles in a background of wild-type DNA (Fig. 2a). Pearson's ( $r$ ) value for the four detected mutation types statistically validated the excellent assay linearity and reproducibility (Fig. 2b). In addition, this method was applied to detect mutations in a large series of FFPE tissues and compared with dideoxy sequencing; the results 
Table 4 Correlation of KRAS mutations with clinicopathological features of CRC patients

\begin{tabular}{|c|c|c|c|c|c|c|c|}
\hline \multirow[t]{2}{*}{ Variables } & \multirow[t]{2}{*}{ Number (\%) } & \multicolumn{2}{|l|}{ KRAS } & \multicolumn{2}{|l|}{ Univariate } & \multicolumn{2}{|l|}{ Multivariate } \\
\hline & & Mutant (\%) & Wild-type (\%) & Odds ratio (95\% Cl) & $p$ value & Odds ratio $(95 \% \mathrm{Cl})$ & $p$ value \\
\hline \multicolumn{8}{|l|}{ Gender } \\
\hline Male & 657 (59.78) & $233(35.46)$ & $424(64.54)$ & Ref. & & Ref. & \\
\hline Female & $442(40.22)$ & $204(46.15)$ & $238(53.85)$ & $1.56(1.22-1.99)$ & $<0.001$ & $1.64(1.26-2.12)$ & $<0.001$ \\
\hline \multicolumn{8}{|l|}{ Age (years) } \\
\hline$\leq 50$ & $232(21.11)$ & $41(17.67)$ & $191(82.33)$ & Ref. & & Ref. & \\
\hline$>50$ & 867 (78.89) & $396(45.67)$ & $471(54.33)$ & $3.92(2.72-5.63)$ & $<0.001$ & $4.17(2.85-6.10)$ & $<0.001$ \\
\hline \multicolumn{8}{|l|}{ Sub-localization } \\
\hline Rectum & 371 (33.76) & 139 (37.47) & $232(62.53)$ & Ref. & & Ref. & \\
\hline Prioximal colon & 373 (33.94) & 155 (41.55) & $218(58.45)$ & $1.19(0.88-1.59)$ & 0.254 & $0.85(0.62-1.17)$ & 0.333 \\
\hline Distal colon & $355(32.30)$ & $143(40.28)$ & $212(59.72)$ & $1.13(0.84-1.52)$ & 0.437 & $0.95(0.69-1.31)$ & 0.754 \\
\hline \multicolumn{8}{|l|}{ Dukes' stage } \\
\hline$A+B$ & $614(56.23)$ & 244 (39.74) & $370(60.26)$ & Ref. & & Ref. & \\
\hline$C+D$ & $478(43.77)$ & $192(40.17)$ & $286(59.83)$ & $1.02(0.80-1.30)$ & 0.886 & $0.99(0.77-1.29)$ & 0.963 \\
\hline \multicolumn{8}{|l|}{ Pathology } \\
\hline Non-ADC & $54(4.91)$ & $10(18.52)$ & $44(81.48)$ & Ref. & & Ref. & \\
\hline ADC & $1045(95.09)$ & $427(40.86)$ & $618(59.14)$ & $3.04(1.51-6.11)$ & 0.002 & $2.41(1.12-5.17)$ & 0.024 \\
\hline \multicolumn{8}{|l|}{ Differentiation } \\
\hline Well & $78(7.10)$ & $23(29.49)$ & $55(70.51)$ & Ref. & & Ref. & \\
\hline Moderate & 752 (68.43) & $310(41.22)$ & 442 (58.78) & $1.68(1.01-2.79)$ & 0.046 & $1.10(0.62-1.96)$ & 0.742 \\
\hline Poor & $256(23.29)$ & $101(39.45)$ & 155 (60.55) & $1.56(0.90-2.69)$ & 0.112 & $1.24(0.66-2.30)$ & 0.503 \\
\hline Unknown & $13(1.18)$ & $3(23.08)$ & $10(76.92)$ & $0.72(0.18-2.85)$ & 0.637 & $0.53(0.13-2.24)$ & 0.388 \\
\hline
\end{tabular}

KRAS V-Ki-ras2 Kirsten rat sarcoma viral oncogene homolog, $C R C$ colorectal cancer, $C$ confidence interval, $A D C$ adenocarcinoma, Ref. reference group

showed a high overall agreement with genotyping, with more mutations detected by the pyrosequencing assay (data not shown).

Dideoxy sequencing and real-time PCR-based techniques are currently the most commonly performed methods for the detection of EGFR and KRAS mutations in the clinic. Direct DNA sequencing has several disadvantages with regard to clinical application [20-22]. The most notable one is its requirement for a DNA template with a relatively high quality. Moreover, it has a relatively low sensitivity, as also revealed by this study (Additional file 1: Figures S2-S5). The real-time PCRbased approaches, such as the amplification refractory mutation system, peptide nucleic acid/locked nucleic acid clamp PCR, and coamplification at lower denaturation temperature PCR have been reported to be more sensitive and detect as low as $1 \%$ mutated alleles in samples containing a mixture of tumor and normal cells $[16,22]$; however, the results obtained are restricted to the screening of mutant versus wild-type tumors and lack any further characterization. In contrast, pyrosequencing could detect all of the mutations within the amplified region, show DNA sequences around targeted nucleotide(s), and provide the quality assurance measurement that is especially important in clinical settings [21, 39]. In this study, our designed pyrosequencing assay using a novel dispensation order could detect and characterize both classical and uncommon EGFR and KRAS mutations (Additional file 1: Figures S1, S6, and S7). In addition, most of the types of degraded DNA extracted from NSCLC- or CRC-FFPE tissues could be used as the pyrosequencing template directly, without requiring further dilution or normalization. Thus, compared with other techniques, the pyrosequencing assay described herein is relatively simple, reliable, and fast. Although real-time PCR-based approaches seem to be more sensitive than our modified pyrosequencing assay, the sensitivity for the detection of mutations by this method is sufficient as it could detect mutations in samples containing as few as $2 \%$ mutated cancer cells (Fig. 2a).

There were some limitations in this retrospective study. First, we had no data on survival analysis. Second, we were unable to demonstrate the intra-patient variability of the EGFR or KRAS mutations between primary lesions and metastatic lesions, because the tests for EGFR and KRAS mutations were performed in either the primary tumor or metastatic tumor sample from each patient. Despite these limitations, using this novel 
pyrosequencing method in routine clinical practice provided a large-scale screening that included unselected Asian NSCLC and CRC patients. Furthermore, it demonstrated the prevalence of EGFR and KRAS mutations and mutation patterns in a Chinese population.

\section{Conclusion}

In summary, our findings suggest that the best clinical independent predictive factors for targeted therapy of Chinese NSCLC patients with EGFR inhibitors include an ADC histology, a nonsmoking history, and a younger age $(\leq 65)$; for Chinese CRC patients, those who are female, older ( $>50$ year old), and have ADC histology may benefit from mAb-based molecularly targeted therapies. Moreover, our designed pyrosequencing method using a novel nucleotide dispensation order is a practical and reliable method for the detection of NSCLC EGFR and CRC KRAS mutations in FFPE samples. The sensitivity, accuracy, and simplicity of the procedure are suitable for genetic testing of NSCLC and CRC patients at the clinical laboratory level.

\section{Additional file}

Additional file 1: Table S1. Molecular epidemiological status of EGFR in NSCLC and KRAS mutation in CRC; Table S2. Characteristics of the human cell lines used for validation and sensitivity testing of the pyrosequencing analysis for EGFR and KRAS mutation detection; Table S3. Characteristics of the known mutant human NSCLC- and CRC-FFPE tissues used for validation of the designed pyrosequencing analysis; Table S4. Primer sequences; Table S5. Actual/theoretical percentages of mutant alleles at given dilutions of EGFR exon 19 c.2235_2249del15 (G>A) (H1650) and exon 21 c.2573T>G (H1975); Table S6. Actual/Theoretical Percent Mutant Allele at Given Dilutions of KRAS exon 2 c.35 G>T (SW480) and C.38 G>A (DLD-1); Figure S1. Nucleotide sequences and novel dispensation order for EGFR exons 18, 19, 20, and 21 mutation analysis as well as KRAS exon 2 mutational analysis; Figure $\mathbf{S 2}$. Analytical sensitivity for mutation detection of homozygous KRAS exon 2 c.35G >T on three consecutive days; Figure S3. Analytical sensitivity for mutation detection of heterozygous KRAS exon 2 c.38G $>$ A on three consecutive days; Figure S4. Analytical sensitivity for in-frame deletion mutation detection of homozygous EGFR exon 19 c.2235_2249 del 15 (G>A) on three consecutive days; Figure S5. Analytical sensitivity for mutation detection of heterozygous EGFR exon 21 c.2573T>G on three consecutive days; Figure S6.

Representative results of EGFR exons 19-21 pyrosequencing analysis

in FFPE samples; Figure S7. Representative results of pyrosequencing KRAS analyses of exon 2 codons 12/13 in FFPE samples.

\section{Competing interests}

The authors declare that they have no competing interests.

\section{Authors' contributions}

GX, FX, XY and YM performed the experiments. GX and FX analyzed the data and drafted the manuscript. YX and LL provided material support. LX participated in the sequence alignment. PW participated in the design of the study and performed the statistical analysis. LS and MY conceived of the study and participated in its designation and helped to draft the manuscript. All authors read and approved the final manuscript.

\section{Acknowledgments}

This study was supported by grants from the National Natural Science Foundation of China (No. 81101848 and No. 81201768).

\section{Author details}

${ }^{1}$ Department of Clinical Laboratory, Xinhua Hospital, Shanghai Jiao Tong University School of Medicine, 1665 Kong Jiang Road, Shanghai 200092, China. ${ }^{2}$ Department of General Surgery, Xinhua Hospital, Shanghai Jiao Tong University School of Medicine, Shanghai 200092, China. ${ }^{3}$ Department of Anorectal Surgery and Colorectal Cancer Center, Xinhua Hospital, Shanghai Jiao Tong University School of Medicine, 1665 Kong Jiang Road, Shanghai 200092, China.

Received: 16 March 2015 Accepted: 2 June 2015

Published online: 18 June 2015

\section{References}

1. Jemal A, Bray F, Center MM, Ferlay J, Ward E, Forman D. Global cancer statistics. CA Cancer J Clin. 2011;61:69-90.

2. Kim HR, Lee SY, Hyun DS, Lee MK, Lee HK, Choi CM, et al. Detection of EGFR mutations in circulating free DNA by PNA-mediated PCR clamping. J Exp Clin Cancer Res. 2013;32:50.

3. Xu JM, Liu XJ, Ge FJ, Lin L, Wang Y, Sharma MR, et al. KRAS mutations in tumor tissue and plasma by different assays predict survival of patients with metastatic colorectal cancer. J Exp Clin Cancer Res. 2014;33:104.

4. Allegra CJ, Jessup JM, Somerfield MR, Hamilton SR, Hammond EH, Hayes DF, et al. American Society of Clinical Oncology provisional clinical opinion: testing for KRAS gene mutations in patients with metastatic colorectal carcinoma to predict response to anti-epidermal growth factor receptor monoclonal antibody therapy. J Clin Oncol. 2009;27:2091-6.

5. Keedy VL, Temin S, Somerfield MR, Beasley MB, Johnson DH, McShane LM, et al. American Society of Clinical Oncology provisional clinical opinion: epidermal growth factor receptor (EGFR) Mutation testing for patients with advanced non-small-cell lung cancer considering first-line EGFR tyrosine kinase inhibitor therapy. J Clin Oncol. 2011;29:2121-7.

6. Yamaguchi N, Vanderlaan PA, Folch E, Boucher DH, Canepa HM, Kent MS, et al. Smoking status and self-reported race affect the frequency of clinically relevant oncogenic alterations in non-small-cell lung cancers at a United States-based academic medical practice. Lung Cancer. 2013;82:31-7.

7. Rosell R, Moran T, Queralt C, Porta R, Cardenal F, Camps C, et al. Screening for epidermal growth factor receptor mutations in lung cancer. N Engl J Med. 2009;361:958-67.

8. Reinersman JM, Johnson ML, Riely GJ, Chitale DA, Nicastri AD, Soff GA, et al. Frequency of EGFR and KRAS mutations in lung adenocarcinomas in African Americans. J Thorac Oncol. 2011;6:28-31.

9. Shi Y, Au JS, Thongprasert S, Srinivasan S, Tsai CM, Khoa MT, et al. A prospective, molecular epidemiology study of EGFR mutations in Asian patients with advanced non-small-cell lung cancer of adenocarcinoma histology (PIONEER). J Thorac Oncol. 2014;9:154-62.

10. Kim TJ, Park CK, Yeo CD, Park K, Rhee CK, Kim J, et al. Simultaneous diagnostic platform of genotyping EGFR, KRAS, and ALK in 510 Korean patients with non-small-cell lung cancer highlights significantly higher ALK rearrangement rate in advanced stage. J Surg Oncol. 2014;110:245-51.

11. Tokumo M, Toyooka S, Kiura K, Shigematsu H, Tomii K, Aoe M, et al. The relationship between epidermal growth factor receptor mutations and clinicopathologic features in non-small cell lung cancers. Clin Cancer Res. 2005;11:1167-73.

12. Itrat M, Essam A, Bassim J. KRAS mutations: does ethnicity play a role [abstract]? J Clin Oncol. 2014;32:e14628.

13. Bisht S, Ahmad F, Sawaimoon S, Bhatia S, Das BR. Molecular spectrum of KRAS, BRAF, and PIK3CA gene mutation: determination of frequency, distribution pattern in Indian colorectal carcinoma. Med Oncol. 2014;31:124.

14. Watanabe T, Yoshino T, Uetake H, Yamazaki K, Ishiguro M, Kurokawa T, et al. KRAS mutational status in Japanese patients with colorectal cancer: results from a nationwide, multicenter, cross-sectional study. Jpn J Clin Oncol. 2013:43:706-12.

15. Wu Y, Lin J, Wang K, Xu C, Zhou Q, Chen G, et al. EGFR mutations in lung cancers and sensitivity to gefitinib in Chinese. J Clin Oncol. 2005;23:7089.

16. Lai $Y$, Zhang Z, Li J, Sun D, Zhou Y, Jiang T, et al. EGFR mutations in surgically resected fresh specimens from 697 consecutive Chinese patients with non-small cell lung cancer and their relationships with clinical features. Int J Mol Sci. 2013;14:24549-59.

17. Liu $Y$, Wu BQ, Zhong HH, Hui P, Fang WG. Screening for EGFR and KRAS mutations in non-small cell lung carcinomas using DNA extraction by 
hydrothermal pressure coupled with PCR-based direct sequencing. Int J Clin Exp Pathol. 2013;6:1880-9.

18. Liao W, Liao Y, Zhou JX, Xie J, Chen J, Huang W, et al. Gene mutations in epidermal growth factor receptor signaling network and their association with survival in Chinese patients with metastatic colorectal cancers. Anat Rec (Hoboken). 2010;293:1506-11.

19. Mao C, Zhou J, Yang Z, Huang Y, Wu X, Shen H, et al. KRAS, BRAF and PIK3CA mutations and the loss of PTEN expression in Chinese patients with colorectal cancer. PLoS One. 2012;7:e36653.

20. Ma ES, Wong CL, Law FB, Chan WK, Siu D. Detection of KRAS mutations in colorectal cancer by high-resolution melting analysis. J Clin Pathol. 2009;62:886-91.

21. Ogino S, Kawasaki T, Brahmandam M, Yan L, Cantor M, Namgyal C, et al. Sensitive sequencing method for KRAS mutation detection by Pyrosequencing. J Mol Diagn. 2005;7:413-21.

22. Ono A, Kenmotsu H, Watanabe M, Serizawa M, Mori K, Imai H, et al. Mutant allele frequency predicts the efficacy of EGFR-TKIs in lung adenocarcinoma harboring the L858R mutation. Ann Oncol. 2014;25:1948-53.

23. Ronaghi $\mathrm{M}$, Uhlen $\mathrm{M}$, Nyren $\mathrm{P}$. A sequencing method based on real-time pyrophosphate. Science. 1998;281:363-5.

24. Stigt JA, tHart NA, Knol AJ, Uil SM, Groen HJ. Pyrosequencing analysis of EGFR and KRAS mutations in EUS and EBUS-derived cytologic samples of adenocarcinomas of the lung. J Thorac Oncol. 2013;8:1012-8.

25. Yan JB, Zhang R, Xiong $C, H u C$, LV Y, Wang CR, et al. Pyrosequencing is an accurate and reliable method for the analysis of heteroplasmy of the A3243G mutation in patients with mitochondrial diabetes. J Mol Diagn. 2014;16:431-9.

26. Sahoo R, Harini W, Babu VC, Patil Okaly GV, Rao S, Nargund A, et al. Screening for EGFR mutations in lung cancer, a report from India. Lung Cancer. 2011;73:316-9.

27. Dogan $S$, Shen R, Ang DC, Johnson ML, D'Angelo SP, Paik PK, et al. Molecular epidemiology of EGFR and KRAS mutations in 3,026 lung adenocarcinomas: higher susceptibility of women to smoking-related KRAS-mutant cancers. Clin Cancer Res. 2012;18:6169-77.

28. Sun YH, Fang R, Gao B, Han XK, Zhang JH, Pao W, et al. Comparable rate of EGFR kinase domain mutation in lung adenocarcinomas from Chinese male and female never-smokers. Acta Pharmacol Sin. 2010;31:647-8.

29. Gao B, Sun Y, Zhang J, Ren Y, Fang R, Han X, et al. Spectrum of LKB1, EGFR, and KRAS mutations in chinese lung adenocarcinomas. J Thorac Oncol. 2010;5:1130-5.

30. Kosaka T, Yatabe Y, Endoh H, Kuwano H, Takahashi T, Mitsudomi T. Mutations of the epidermal growth factor receptor gene in lung cancer: biological and clinical implications. Cancer Res. 2004;64:8919-23.

31. Tanaka T, Matsuoka M, Sutani A, Gemma A, Maemondo M, Inoue A, et al. Frequency of and variables associated with the EGFR mutation and its subtypes. Int J Cancer. 2010;126:651-5.

32. Zhang Y, Sun Y, Pan Y, Li C, Shen L, Li Y, et al. Frequency of driver mutations in lung adenocarcinoma from female never-smokers varies with histologic subtypes and age at diagnosis. Clin Cancer Res. 2012;18:1947-53.

33. Kim Y, Hammerman PS, Kim J, Yoon JA, Lee Y, Sun JM, et al. Integrative and comparative genomic analysis of lung squamous cell carcinomas in East Asian patients. J Clin Oncol. 2014;32:121-8.

34. Chougule A, Prabhash K, Noronha V, Joshi A, Thavamani A, Chandrani P, et al. Frequency of EGFR mutations in 907 lung adenocarcioma patients of Indian ethnicity. PLoS One. 2013;8:e76164.

35. Kenmotsu H, Serizawa M, Koh Y, Isaka M, Takahashi T, Taira T, et al. Prospective genetic profiling of squamous cell lung cancer and adenosquamous carcinoma in Japanese patients by multitarget assays. BMC Cancer. 2014;14:786.

36. Vaughn CP, Zobell SD, Furtado LV, Baker CL, Samowitz WS. Frequency of KRAS, BRAF, and NRAS mutations in colorectal cancer. Genes Chromosomes Cancer. 2011;50:307-12.

37. Neumann J, Zeindl-Eberhart E, Kirchner T, Jung A. Frequency and type of KRAS mutations in routine diagnostic analysis of metastatic colorectal cancer. Pathol Res Pract. 2009;205:858-62.

38. Gil Ferreira C, Aran V, Zalcberg-Renault I, Victorino AP, Salem JH, Bonamino $\mathrm{MH}$, et al. KRAS mutations: variable incidences in a Brazilian cohort of 8,234 metastatic colorectal cancer patients. BMC Gastroenterol. 2014;14:73.

39. Imamura Y, Lochhead P, Yamauchi M, Kuchiba A, Qian ZR, Liao X, et al. Analyses of clinicopathological, molecular, and prognostic associations of KRAS codon 61 and codon 146 mutations in colorectal cancer: cohort study and literature review. Mol Cancer. 2014;13:135.

\section{Submit your next manuscript to BioMed Central and take full advantage of:}

- Convenient online submission

- Thorough peer review

- No space constraints or color figure charges

- Immediate publication on acceptance

- Inclusion in PubMed, CAS, Scopus and Google Scholar

- Research which is freely available for redistribution

Submit your manuscript at www.biomedcentral.com/submit 